\title{
The challenge of rationality in computer access for the visually impaired
}

\author{
ROBERT M. LAMBERT \\ Concordia University, Montreal, Quebec, Canada
}

\begin{abstract}
Problems encountered by the visually impaired in accessing computers are discussed from a blind Apple user's point of view. Difficulties arising from hardware, software, and inadequacies of documentation are considered. Particular attention is paid to access limitations involving paperless Braille and synthetic speech output. Avenues of solution for the problems are suggested. The main remedies proposed are increasing standardization of software design and interfacing requirements, along with greater producer-consumer consultation.
\end{abstract}

I remember well sitting on my mother's lap as a blind child about 6 years old and hearing her say "some day there will be a machine with which you'll be able to read print just like I do." Nonetheless encouraged, I grew up through the 1940s and 1950s more the witness to a selfimposed skepticism than the beneficiary of my mother's optimism. It was clear that my mother's faith in emerging technology referenced much more than the presentday OPTACON. She believed that my missing visual functions would, at least in the long term, be replaced or compensated for in their entirety by new and wonderful technological devices. Although I was tantalized by this idea, it seemed to be a prodigious economic impracticality.

In a real sense, I was right. Governments at all levels have displayed a remarkably persistent reluctance to invest in the development and marketing of technological aids for the visually impaired. Private nonprofit corporations with such intentions have, in general, failed to flourish. The profit-oriented corporate giants have indeed found the very limited size of the visually impaired market an adequate reason for failing to develop or produce devices well within the scope of their development and production capability.

In an equally real sense, I was wrong. I did not foresee the bold venturesome creation of small new primary companies that would seek to make an honest and reasonable profit by developing and selling technology to blind people. Nor did I anticipate the growth potential for secondary industries that might specialize in developing peripheral devices and software to facilitate the use of modern computers by the visually impaired.

I might almost feel a little embarrassed to write the present paper. As I revel in my ability to type it on a standard commercially available home computer, using an inexpensive speech synthesizer and a specially written word processing program that effectively exploits the speech

The author's mailing address is: Psychology Department, Concordia University, Loyola Campus, 7141 Sherbrooke St. West, Montreal, Quebec H4B 1R6, Canada. synthesizer, I am making critical observations of the very technology that ensures my revelry. Yet, on the other hand, these critical observations are not the result of ingratitude. They represent, instead, the desire of a "walking blind computer user" to "run." Even more, they reflect my belief that running with the computer is possible for a blind user if those of us concerned with access to technology for the visually impaired can provide a rational analysis of current access problems and a concerted effort to solve them. Thus, in the next section, I briefly outline and, where feasible, illustrate some of the problems of device operation, software, and documentation that have imposed unnecessary limitations upon access to computers by the blind. In the final section of the paper, I suggest some consultative and cooperative ways in which such problems might be addressed and solved.

\section{PROBLEMS OF COMPUTER ACCESS BY THE VISUALLY IMPAIRED}

In this section of the paper, I discuss two kinds of problems that limit a blind user's access to computers. First, I discuss problems in the operation of access equipment. Then I discuss problems inherent in software and documentation. In each case, I characterize the limitations generally and illustrate them with at least one specific example. Examples will be chosen so as to be typical of others that might have been presented in their stead. While my personal experience has been largely limited to the use of Apple microcomputers, I have selected the problems and illustrations on the basis of considerable discussion with other blind computer users, and I trust that they are indicative of those encountered in the use of other types of microcomputers.

\section{Problems of Equipment Operation}

Access-limiting problems of equipment operation fall into two broad categories. On one hand, there are characteristics of access equipment design that accelerate equipment deterioration. These are exemplified by overly delicate switches or cable connectors that defy efficient 
utilization by blind equipment operators. On the other hand, there are inefficient design characteristics inherent in equipment firmware or operating systems that painfully complicate and slow down an operator's work. These are exemplified by undue redundancies in accessing disk directories.

Frequent use of the Apple IIe computer for purposes such as the development of Pascal programs or the editing of Wordstar text files requires access to the monitor screen via the OPTACON. Although the OPTACON's computer lens module is fitted with nylon ball bearings to allow smooth and benevolent movement around the screen, these ball bearings cannot withstand long-term extensive employment. As little as 1 year of concentrated use reduces these protective elements to flattened intransigents. The result is a monitor screen that becomes scratched to the point of OPTACON unreadability. The truly frustrating aspect of this problem is not merely that it happens, but rather that it lacks a ready solution. The scratched glare filter on the Apple II monitor cannot be economically replaced, and there appears to be no way to replace the damaged ball bearings in the lens module of the OPTACON.

A primary means of accessing the computer by way of Braille output has been the family of VersaBraille devices, now in its second generation (Telesensory Systems, Inc., 455 N. Bernardo Ave., Mountain View, CA 94043). The new disk-based VersaBraille system has corrected many inadequacies found in its ancestor. Yet, curiously, it has also created some new inadequacies all its own.

A major issue in the smooth operation of any interface is the matching of device control parameters. Baud rates for the transfer of information between the connected devices, mutually agreeable data formats, and handshaking protocols must clearly be matched. The older model VersaBraille devices provided for this matching via configuration control parameters that could be defined and stored on magnetic tape. A given set of stored configuration control parameters could easily be loaded into the VersaBraille system and used either to access a telephone modem or other device. Once the operator had defined and stored a set of parameters, he/she needed to remember nothing more than the name of the file in which they had been stored in order to use them.

The new generation VersaBraille device makes the matching of parameters between itself and other equipment considerably more awkward. That there is a vastly larger number of parameters to be set in the new VersaBraille unit indeed adds appreciably to the unit's flexibility. Furthermore, the parameters are grouped under menu access headers in such a way as to give their functions some very useful conceptual clarity. The awkwardness arises from the fact that parameter settings cannot be peripherally stored. Once parameters have been set, the VersaBraille system remembers them until they are manually changed, or until the system is reset. Thus, if a user alternates accesses via the VersaBraille system among a number of different devices, he or she must perhaps remember, and must certainly key into the VersaBraille memory, a whole set of changed parameters for each new accessed device. Clearly this requirement is retrogressive and unduly complicates a blind user's access to any kind of technology that interfaces with the VersaBraille system.

\section{Problems of Software and Documentation}

Preemptive programming. Computer programmers and users of computers share a common sense of frustration when a program crashes. This sense of frustration is greatly magnified when the crash results from an error or an indiscretion in program use. To reduce the risk of such crashes, programmers frequently resort to painstaking methods of "idiot proofing" their products. Inappropriate user responses to prompts are trapped before their digestion by a program leads to an $1 / O$ message accompanied by a program termination. Redirection of output from the video screen to peripheral devices, such as printers, is handled by the program automatically, to avoid user-instigated mistakes in the effort to accomplish it.

Occasionally, but nonetheless vexatiously, the zeal with which a commercial applications program is idiot proofed denies a blind computer user some needed flexibility in the use of the program. Generally, the serial interface between an Apple computer and a VersaBraille unit is accomplished by way of a serial card placed in slot 2 of the Apple's peripheral board. If a program is run on the Apple under either DOS 3.3 or under PRODOS, its output can be sent to the VersaBraille unit only by the issuance of a $<\mathrm{PR} \# 2>$ command, either from the operating system or from within the program itself. If the applications program is written in BASIC, and contains no machine language code that preemptively assigns output to the video screen (slot 0 ), then there is no difficulty in directing output to the VersaBraille system. On the other hand, I have encountered several commercial programs in which such preemptive strikes against access by a blind user are found. In these cases, the user may issue a $<P R \# 2>$ command from DOS only to find, to his/her dismay, that when the program begins execution, his/her desire and effort on behalf of VersaBraille output are frustrated. Even worse than the frustration of programs that prevent output to the VersaBraille system is the truly irritating fact that although many programs running under PRODOS can send output to that device, much of PRODOS itself cannot.

Synthetic speech problems. A number of speech synthesizers available for costs around $\$ 200$ have, for several years, offered visually impaired computer users the lure of applications programs with spoken word output. Although a wide variety of software packages does, indeed, fulfill this promise, significant problems continue to trouble aural access to computers.

Algorithms to convert ASCII characters into intelligible speech must be large and relatively complex. If those algorithms are stored in the limited memory of a microcomputer, there is a serious risk of encountering at least one of two difficulties. First, the internal data space avail- 
able for use by data-intensive programs may be severely constrained by the memory requirements of the speech algorithms. Second, and more importantly, applications programs written in machine language often overwrite the speech algorithms that are prestored in the memory locations that such programs utilize. Thus commercially viable programs, such as the Lotus series, Apple Works, and the like, are generally not accessible to blind computer users through spoken word output.

In a valiant attempt to solve these problems, a number of specialized software companies have sprung up to write or modify commercial programs for use with inexpensive speech synthesizers. I wish in no way to diminish the efforts of these companies. Many of their products have given visually impaired computer users some access to computer applications that otherwise would have likely remained totally inaccessible. Yet, with few exceptions, specially developed software designed to use synthetic speech clearly lacks the power and scope of commercial packages available to the normal user. I know of nothing that talks and is analogous to Visicalc. Nor do I know of any talking software that is comparable to the Locksmith series of programs. Furthermore, most of the companies that produce specialized software that "talks" to the visually impaired are staffed by only one or two skilled programmers. Thus, a somewhat less than truly professional job of debugging programs is fairly common. Finally, there is at best a very limited amount of premarket field testing of such programs to ensure their freedom from troublesome bugs.

The BEX program, produced by Raised Dot Computing (408 S. Baldwin, Madison, WI 53703) exemplifies both the best features of specially developed software and some of its problems. BEX is a combination word processing and $\mathrm{I} / \mathrm{O}$ program. It allows input via the Apple keyboard, the VersaBraille system, an optical scanner such as the Kurzweil reading machine (Kurzweil Computer Products, 33 Cambridge Parkway, Cambridge, MA 02142), or a standard DOS 3.3 text file. It permits output to an Echo+ speech synthesizer, several graphically produced large-print video screen formats, a standard Apple video display, a VersaBraille system, a DOS 3.3 TEXTFILE, or a printer. Output to a printer can be produced either in standard ink print, large print, or Braille. The program is capable of translating ASCII coded characters into grade $\$ 2$ Braille, so that Braille output can appear in contracted form. The word processing and printed display formatting options of BEX are very powerful. Yet, despite constant updating of the BEX program, it remains a comfortable home for several varieties of bugs. Although its manual bravely announces its ability to place a page header on any of the page lines 1 through 3 , these headers are placed on line 1 of the page no matter what the user requests. Sometimes, during the synthetic speech of large blocks of text within the edit function of the program, long lists of numbers and other garbage not entered into the text by the user are glibly recited. Occasionally, strange numbers precede the appearance of text entered by the user in an ink print printout from the program.

A more generic limitation of access to computers by visually impaired users is also found in the software that drives inexpensive speech synthesizers. This limitation inheres in the fact that the software is loaded from floppy disks and is thus operating system dependent. While the Street Electronics Echo+ (Street Electronics, 1140 Mark Ave., Carpentaria, CA 93013) automatically captures and speaks the contents of a "PRINT" statement in an Applesoft Basic program run on the Apple computer, it cannot automatically speak the analogous contents of a Pascal "WRITELN" statement. The Echo+ software can be run under the control of Apple DOS 3.3 or PRODOS, but it cannot run under the control of Apple Pascal 1.1 or 1.2. Nor can it run under the control of the Apple CP/M operating system, even if the Apple computer is equipped with a Z80 processor card. Consequently, valuable commercial programs such as Wordstar, Dbase 2, and Aladdin, cannot be accessed by blind users on the Apple via the Echo+ speech synthesizer. Although this limitation in the use of synthetic speech is important, in fairness to the producers of many speech synthesizers, another word should be said. Software is often available from manufacturers that may allow a skilled visually impaired program developer to generate speech output from a Pascal program of his/her own authorship.

Documentation. Problems of documentation again fall into several categories. I shall briefly mention three types of difficulty.

Even for those commercial programs whose output is accessible to a visually impaired computer user, practical use of the programs is often at best tedious and risky because manuals explaining their use are inaccessible. Specific publishers of Braille and/or recorded material for the blind, such as the Howe Press (88 St. Stephen Street, Boston, MA), may obtain copyrights from software publishers and produce accessible manuals for the visually impaired in instances where demand is great. Thus, today it is possible to obtain accessible manuals for Applesoft BASIC. But the market for particular applications programs is fractionated and, hence, so too is the market for their manuals. In this context, specific publishers of Braille or recorded material for the blind do not generally undertake to make manuals available.

An example of another problem is once again provided in the framework of inexpensive speech output. Available manuals, whether directly accessible to the visually impaired or not, frequently fail to provide information in sufficient technical detail to optimize the functioning of a visually impaired user. Currently, disk-based text files that can be "read out" by the Echo+ speech synthesizer can adequately instruct a blind Apple computer user how to produce usable spoken word output on the Echo+ from congenial applications programs. But adaptation of the Echo+ software for use in Pascal programs developed by 
a visually impaired user is explained nowhere in the Echo+ documentation. Construction and use of Apple Pascal "library units" is left entirely to the Apple Pascal language programming documentation, which is inaccessible to the visually impaired. Construction of Pascal source code for "talking" the contents of numerical and character variables in Pascal programs is wholly a function of the visually impaired user's programming initiative.

Some of the most critical problems of documentation occur with respect to the interface between computers and other devices. These problems are critical, because in addition to causing confusion for the visually impaired user, they may also be expensive. In general, they relate to ambiguities, inconsistencies, and inadequacies in the specification of interfacing boards, connectors, and parameters. Furthermore, they are most common in situations requiring a serial interface.

A very meaningful illustration of problems in the documentation about serial interfacing comes from Raised Dot Computing's BEX program interface manual. Many important uses of BEX require that an Apple computer be serially connected to some external piece of equipment. Thus, the producers of BEX have taken appreciable care to spell out in detail how an Apple Super Serial board should be used to achieve a good interface. A problem arises because the Apple computer may sometimes need to operate as a DTE device, (Data Terminal Equipment RS232C; serial output on pin 2, input on pin 3), while at other times it either can or must operate as a DCE device (Data Communications Equipment RS232C; serial output on pin 3, input on pin 2).

A "jumper block" on the Apple Super Serial board can be plugged in either so as to wire the board as a DTE unit or so as to wire it as a DCE. Two banks of dip switches on the board are set differently to establish baud rate, data format, handling of interrupts, display format, and handshaking parameters according to whether the board is DTE or DCE. The BEX interface manual prescribes a DCE board with clearly assigned dip switch settings to interface BEX with a VersaBraille system. On the other hand, use of the Apple computer with a telephone modem and modem software requires a DTE serial board with generally different switch settings. Thus the visually impaired user who wishes to work sometimes with BEX and sometimes with a modem has to make regular and awkward physical changes in the configuration of his/her serial board, or, more plausibly, he/she has to purchase two separate serial boards, wiring one DTE and the other DCE. The latter solution costs the user a fairly large amount of scarce money-and all of this is avoidable. The writer has found that a single serial board, wired DTE and having a standard pattern of switch settings, can in fact be used for both purposes.

I do not believe that the problems discussed in this section are inevitable. Many of them can be solved, or at least ameliorated, by better use of consultative resources and good planning. In the next section, I discuss these possibilities briefly.

\section{SOME THOUGHTS ABOUT PROBLEM SOLUTIONS}

A thoughtful examination of the problems discussed thus far in this paper suggests two major causes. First, the providers of access to computers for the visually impaired are, by and large, small cottage type producers with budgets inadequate to maintain skilled staffs of competent programmers and technical writers. Second, these producers develop and market their products in competitive isolation, both from one another and from the consumer whose needs they seek to serve. In the most general sense, then, solutions must rest upon two main efforts. The pool of talent responsible for access system development must be made larger, and consultation among producers and consumers must be expanded. A detailed mechanism for realizing these efforts is well beyond the scope of this paper. What I shall attempt, therefore, is to identify some of the resources available to them and some of their ideal objectives.

As the sheer number of access devices and accessible programs available to the visually impaired has increased over the past 10 years; so too has the zeal with which visually impaired people have committed themselves to using computers. In both residential schools for the blind and mainstream educational programs serving them, courses in the use of computers and devices for accessing them have proliferated. Computer training courses for the adult visually impaired have appeared and flourished in many rehabilitation settings and in universities. Visually impaired individuals are entering the computer programming profession in ever growing numbers. Indeed, it is reasonable to think that, among visually impaired computer users, the level of computer literacy may be generally higher than it is among nonimpaired users.

The relatively large proportion of visually impaired people who have become seriously involved in computer use has led to the development of several structured organizations with creative interests in the use of computers by the visually impaired. The American Council of the Blind has included for some years a special interest group of Blind Data Processors as a constituent organization. At its 1986 international conference, the Association for the Education and Rehabilitation of the Blind and Visually Impaired established a professional division on Information and Technology largely to promote access to computers for the visually impaired. The Journal of Visual Impairment and Blindness now includes a regular section on access to computers. Supplementing these structured vehicles is a host of modem communications networks and local blind users groups.

Although producers of access devices and software are clearly aware of these organizations as sources of market interest in their products, the often talented member- 
ship has been ignored as a source of assistance and guidance in product development. Whenever and wherever such organizations meet, product vendors are there to exhibit their wares. But rarely, if ever, do the producers' representatives make an effort to tap, first hand, the knowledge and needs of the organizational participants. Yet this body of visually impaired users can test programs and devices under development. They also can serve as potentially very useful proofreaders and constructive critics of software and device manuals. I believe that a coalition of producers working coherently together could significantly enhance the quality of access facilities for the visually impaired by utilizing these resources.

Both communication and the computerization of information processing have reaped tremendous benefits from increasing standardization. Indeed, without an RS232 protocol for serial data transfer or an ASCII code for the numerical encoding of data, contemporary computer access might well be a nightmare of confusions for all of us. There is thus good reason to believe that similar kinds of standardizations could also be of specific assistance in reducing special confusions attending access to computers by the visually impaired. Standards could be considered pertaining to the utilization of RAM by test-to-speech algorithms driving speech synthesizers. Standards could be considered with respect to the data length and format of serial information transferred between computers and access devices. Even the amount and methods of proper idiot proofing for accessible programs could be governed by meaningful standards. To achieve this would again require a coalition of access system producers and a process of consultation with visually impaired computer users.

The formation of producer coalitions to enhance and standardize their products through a user-consultative process is governed partly by a kind of inertia. It requires a sort of promotional initiative to "stop not happening." Although it is certainly unclear from where such an initiative should come, a symposium on problems of access to computers by the visually impaired might well set an appropriate coalition of producers as its terminal objective. Furthermore, since the Association for the Education and Rehabilitation of the Blind and Visually Impaired and the American Foundation for the Blind have obviously committed themselves to a serious concern about such problems, one might hope that the pertinent initiative might come from these organizations. 